\title{
Diagnosing asthma in children or adolescents/young adults? It is time for a change! How timing is everything, also in clinical practice
}

\author{
Peter J. Merkus ${ }^{1}$ and Mariëlle W. Pijnenburg ${ }^{2}$ \\ Affiliations: 'Division of Respiratory Medicine and Allergology, Radboudumc Amalia Children's Hospital, \\ Nijmegen, The Netherlands. ${ }^{2}$ Division of Respiratory Medicine and Allergology, Dept of Paediatrics/Paediatric \\ Respiratory Medicine, Erasmus MC - Sophia Children's Hospital, Rotterdam, The Netherlands.
}

Correspondence: Peter J. Merkus, Division of Respiratory Medicine and Allergology, Radboudumc Amalia Children's Hospital, P.O.Box 9101, 6500 HB Nijmegen, The Netherlands. E-mail: Peter.MerkusQradboudumc.nl

@ERSpublications

It is time to innovate the way we look at (diagnosing) asthma https://bit.ly/38SKy1C

Cite this article as: Merkus PJ, Pijnenburg MW. Diagnosing asthma in children or adolescents/young adults? It is time for a change! How timing is everything, also in clinical practice. Eur Respir J 2020; 56: 2002687 [https://doi.org/10.1183/13993003.02687-2020].

In $1190 \mathrm{AD}$, in one of the first expert-based guidelines on asthma, Maimonides already points out that "asthma should be treated according to the various causes that bring it about." He further states that "one can only manage the disease properly with a thorough knowledge of the patient's constitution and his individual organs, his age and habits, the season and the climate" [1]. So he already recognised several risk factors, such as genetic components, environmental exposures and comorbidities. Since then, we have come a long way, and think we understand and treat asthma better, but we can still struggle to diagnose or rule out asthma in patients who are referred to us. And this is even more of a challenge when we are dealing with (young) children.

After referral of a child with respiratory symptoms we traditionally focus on the severity, frequency and nature of the symptoms on one hand and the medical and family history on the other, incorporating known factors that increase the risk for asthma. Traditionally, the next step is to assess whether functional measurements can be of any help in supporting the diagnosis of asthma. Which test is used, and in which order, depends on factors such as age of the patient, local tradition or preferences, availability and resources. Obviously, in a patient with typical symptoms of allergic asthma with signs of reversible airways obstruction, there is no diagnostic problem. It is the patient with less specific and/or variable respiratory symptoms and function tests who forms a diagnostic challenge.

And in this field, it is time for innovation.

In the past, we used techniques because of availability, ease of use or affordability, not because of solid validation. An interesting example was the peak flow meter, introduced over 50 years ago as a diagnostic and monitoring tool in obstructive lung disease. Because of excellent marketing and low costs, the peak flow meter was a great success and was adopted without too much criticism. Since then, however, we know that peak expiratory flow (PEF) is a very crude and insensitive proxy for peripheral airway obstruction, useful for epidemiological research but not for the individual patient (figures 1 and 2) $[2,3]$. 

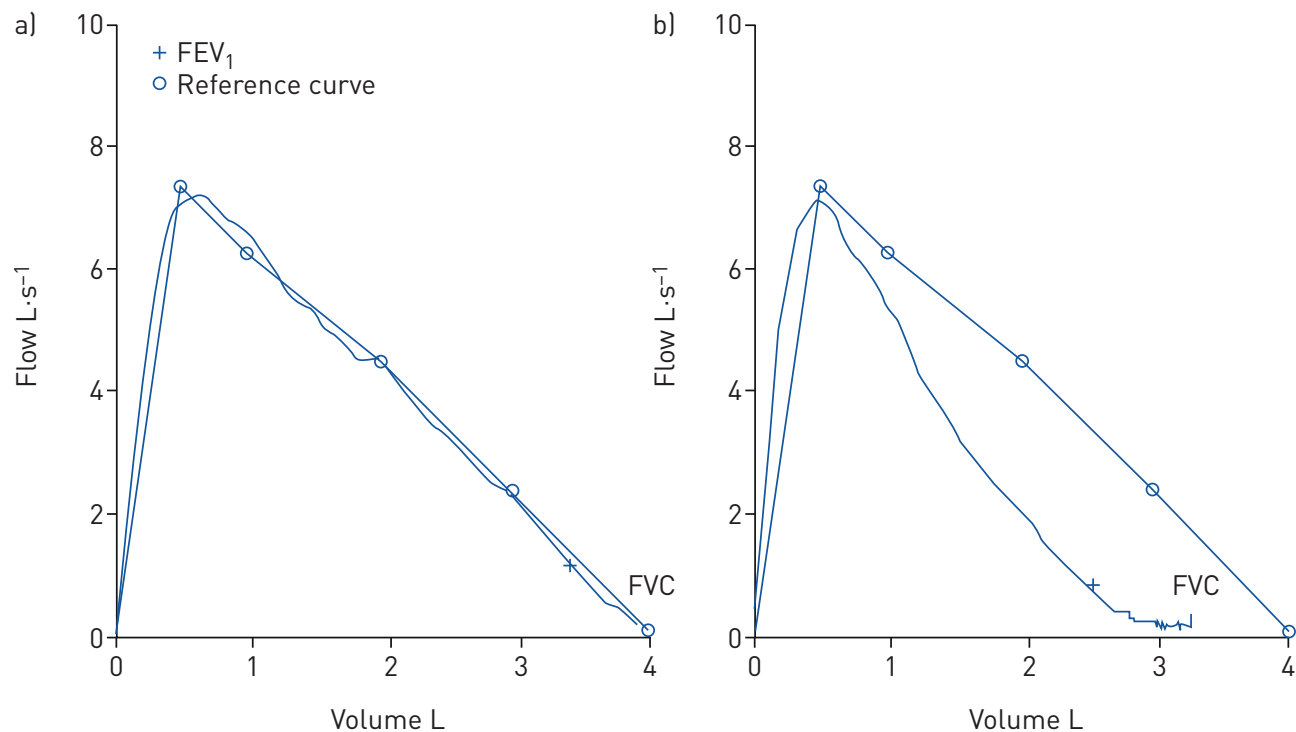

FIGURE 1 Two maximal expiratory flow-volume (MEFV) curves from a child with asthma. a) MEFV curve prior to bronchoprovocation with histamine. b) MEFV curve after clinical recovery of a histamine provocation test. Peak expiratory flow has restored, but forced expiratory volume in $1 \mathrm{~s}\left(\mathrm{FEV}_{1}\right)$ and forced vital capacity $(\mathrm{FVC})$ are still diminished. Note: the loss of 0.5 L FVC indicates airway closure. Reproduced from [2] with permission.

And although it has even been scientifically confirmed that PEF is almost exclusively determined by flow limitation in central airways [4], PEF monitoring is still advised as a diagnostic or monitoring tool for asthma in several current international guidelines [5-7].

So it is useful to critically and structurally analyse which test is most useful in supporting a clinical diagnosis of asthma, using the doctor's diagnosis of asthma by an experienced professional as the gold standard.

Previously, the Bern paediatric asthma group did exactly that and found that specific questions about triggers and severity of wheeze, measurement of fraction of exhaled nitric oxide $\left(F_{\mathrm{ENO}}\right)$ and bronchial provocation testing by methacholine or exercise contributed more to the diagnosis of asthma in 111 school-aged children than spirometry, bronchodilator reversibility and skin-prick tests [8]. However, this
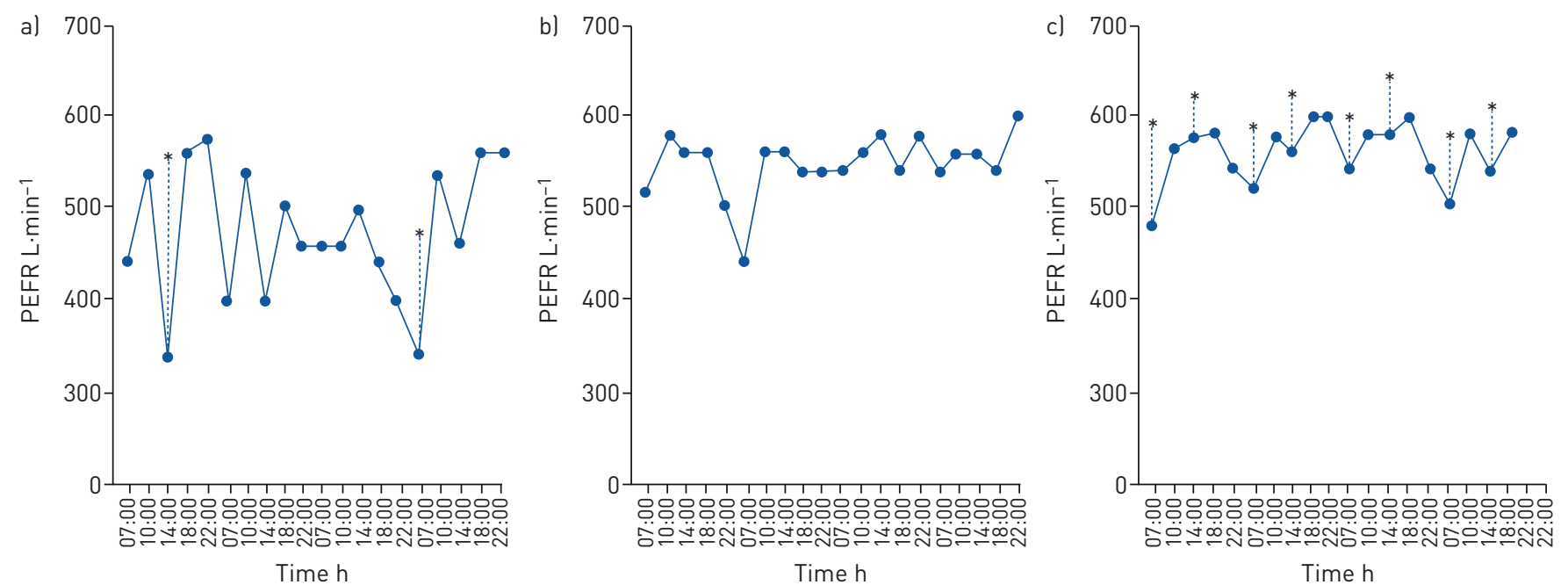

FIGURE 2 Peak expiratory flow rate (PEFR) at 07:00, 10:00, 14:00 and 22:00 h during a) the first 4 days of treatment, b) 4 days of the following week (beclomethasone $0.5 \mathrm{mg}$ twice daily and fenoterol when required), and c) the last 4 days before the fatal attack (beclomethasone 1 mg twice daily and fenoterol $0.4 \mathrm{mg}$ twice daily). The daily variability of PEFR at each respective time-point was: a) 41, 26, 20 and $39 \%$ (mean value $32 \%$ ); b) 14, 21,7 and 10\% (mean value 13\%); and c) 17, 13, 10 and 14\% (mean value 14\%). The reversibility to fenoterol was a) 62 and $35 \%$ (mean value 49\%) and c) 23, 7, 13, 9, 10, 14 and 11\% (mean value 12\%). Dotted lines and asterisks represent reversibility to fenoterol. Reproduced from [3] with permission. 
was carried out in the setting of a tertiary referral clinic with a selected study population with high prevalence of asthma, which explained high positive predictive values. For external validation purposes, DE Jong et al. [9] conducted a study that is published in this issue of the European Respiratory Journal. To assess the usefulness of several tests in children 5-17 years old who were referred by the general physician, a structured diagnostic assessment of respiratory symptoms, diagnostic tests and two paediatric diagnostic algorithms devised by the Global Initiative for Asthma (GINA) and the National Institute for Health and Care Excellence (NICE) were carried out. The gold standard was the diagnosis of definite or probable asthma, or other disease, made by an experienced paediatric pulmonologist based on medical history, clinical examination and all test results. Objective testing included allergy testing, measurements of $F_{\text {ENO}}$, spirometry, body plethysmography and bronchodilator testing (with optional bronchoprovocation testing). The outcome was quite disappointing but clinically relevant: neither single tests nor the algorithms as proposed by GINA and NICE were very helpful in diagnosing asthma in routine clinical care because of low sensitivity (too many children with asthma could not be identified by the standard set of tests).

Therefore, the authors conclude that there is a need for a better diagnostic algorithm to combine respiratory symptoms and objective tests to diagnose asthma. In their paper they did not study the diagnostic value of combining several objective tests, and although a combination is included in the GINA and NICE algorithms, an even better combination may be found and this may be a first step.

We propose that we should start looking at asthma as it is: a condition with highly variable presentation with respect to symptoms, inflammation and airways obstruction, with in between intervals with little or no symptoms and normal airway function.

Hence it is not surprising that the diagnosis of asthma cannot easily be made during a planned visit to the outpatient clinic, when children do not experience their typical complaints or present their typical symptoms.

Therefore, we should innovate the way we look at asthma, ask the patient to cooperate and also be prepared to be more flexible ourselves: 1) we can ask patients (and their parents or partners) to make videos with their smartphone of the typical symptoms, as an aid to clarify the nature and severity of the respiratory symptoms; 2) we should preferably invite patients to visit the outpatient clinic for spirometry and reversibility testing only when they have the typical symptoms that they were referred for; 3 ) occasionally and if available, we could use handheld devices in the home situation that can assess forced expiratory volume in $1 \mathrm{~s}$ rather than PEF and ask for measurements before and after bronchodilation or longitudinal measurements; and 4) our patients spend more than $99.999 \%$ of their time outside the clinic, so e-health applications can facilitate and improve remote diagnostics and monitoring.

But furthermore, asthma is a heterogeneous disease and not all asthma is the same. For instance, asthma-like symptoms may develop both in the presence as well as in the absence of airway inflammation [10]. For the diagnosis of asthma we are still considering asthma as a homogeneous entity, and it might be very refreshing to deconstruct "asthma" into identifiable and treatable traits such as airway phenotype, comorbidities and social and environmental factors, without bothering too much with an exact diagnosis $[11,12]$.

We think future diagnostic asthma guidelines and algorithms should innovate, modernise and choose a more pro-active approach, provide flexibility on timing of diagnostic testing and use smartphones and/or wearables to be able to diagnose or rule out asthma more accurately, and improve monitoring. The new European Respiratory Society task force on diagnosis of asthma in children is timely and we hope this taskforce will come with a pragmatic approach to detect or rule out asthma in a simple, affordable and fast way and with high predictive value.

Conflict of interest: None declared.

\section{References}

$1 \quad$ Rosner F. Moses Maimonides' treatise on asthma. Thorax 1981; 36: 245-251.

2 Roukema J, Gerrits P, Merkus PJ. Airway hyperresponsiveness in children. Eur Respir Monogr 2012; 56: 158-171.

3 Setta M, Thiene G, Crescioli S, et al. Fatal asthma in a young patient with severe bronchial hyperresponsiveness but stable peak flow records. Eur Respir J 1989; 2: 1008-1012.

4 Pedersen OF, Brackel HJ, Bogaard JM, et al. Wave-speed-determined flow limitation at peak flow in normal and asthmatic subjects. J Appl Physiol 1997; 83: 1721-1732.

5 Global Initiative for Asthma. Global Strategy for Asthma Management and Prevention. 2020. https://ginasthma. org/wp-content/uploads/2020/04/GINA-2020-full-report_-final-_wms.pdf Date last accessed: 1 July 2020.

6 BTS-SIGN. British Guideline on the Management of Asthma. www.sign.ac.uk/assets/sign153.pdf Last updated: September 2016. Last accessed: June 2020.

7 NICE. Asthma: Diagnosis, Monitoring and Chronic Asthma Management. https://www.nice.org.uk/guidance/ng80 Date last updated: 29 November 2017. Last accessed: June 2020. 
8 de Jong CCM, Pedersen ESL, Mozun R, et al. Diagnosis of asthma in children: the contribution of a detailed history and test results. Eur Respir J 2019; 54: 1901326.

9 de Jong CCM, Pedersen ESL, Mozun R, et al. Diagnosis of asthma in children: findings from the Swiss Paediatric Airway Cohort. Eur Respir J 2020; 56: 2000132.

10 Fielding SS, Pijnenburg M, de Jongste J, et al. Does treatment guided by exhaled nitric oxide fraction improve outcomes in subgroups of children with asthma? Eur Respir J 2020; 55: 1901879.

11 Pavord ID, Beasley R, Agusti A, et al. After asthma: redefining airways diseases. Lancet 2018; 391: 350-400.

12 Bush A, Fleming L, Saglani S. Severe asthma in children. Respirology 2017; 22: 886-897. 